\title{
Research Progress on the Role of miRNA-448 in Tumor
}

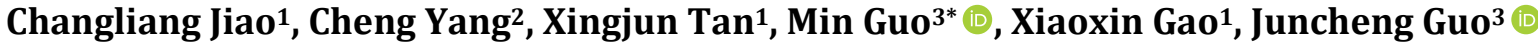 \\ ${ }^{1}$ Hainan Vocational University of Science and Technology, Haikou, China \\ ${ }^{2}$ The First Affiliated Hospital of Hainan Medical College, Haikou, China \\ ${ }^{3}$ Hainan Mental Health Association, Haikou, China \\ Email: ${ }^{\star g} 2002 \mathrm{~m} @ 163 . c o m$
}

How to cite this paper: Jiao, C.L., Yang, C., Tan, X.J., Guo, M., Gao, X.X. and Guo, J.C. (2021) Research Progress on the Role of miRNA-448 in Tumor. Journal of Cancer Therapy, 12, 751-758.

https://doi.org/10.4236/jct.2021.1212065

Received: November 25, 2021

Accepted: December 27, 2021

Published: December 30, 2021

Copyright (c) 2021 by author(s) and Scientific Research Publishing Inc. This work is licensed under the Creative Commons Attribution International License (CC BY 4.0).

http://creativecommons.org/licenses/by/4.0/

(c) (i) Open Access

\begin{abstract}
MicroRNA (miRNA) is a class of endogenous non-coding and regulatory single stranded small RNA molecules, about 18 - 24 nucleotides in length. More than 800 miRNA coding genes have been identified in the human genome, and about 113 target genes are predicted to be regulated, which distinguishes it from most oligonucleotides and functional RNA degraded fragments. It is involved in the regulation of cell proliferation, differentiation, apoptosis and other cell activities. At the same time, it is abnormal in liver cancer, breast cancer, glioma and other tumors, becoming a new biomarker, and participating in cancer differentiation, invasion and metastasis through the interaction with multiple target genes, such as SATB1, NF-KB and 5-HT2B. Mir-448 is abnormally expressed in a variety of tumor cells, such as liver cancer and non-small cell lung cancer. mirNA-448 may play a very important role in tumors. In order to deepen the understanding of the role of mirNA-448 in tumors, this paper reviews the research progress of the role of mirNA-448 in tumors.
\end{abstract}

\section{Keywords}

miRNA, The miRNA-448, Tumor, The Research Progress

\section{Introduction}

MicroRNA (miRNA) is a class of non-coding small RNA molecules consisting of 18 - 24 bases, which are processed by Dicer enzyme by $70-90$ bases of single-stranded RNA precursors with hairpin structure. By binding to target gene mRNA 3'-UTR, miRNA inhibits the translation of target genes or degrades them, thus inhibiting target gene expression [1]. Studies have shown that human 
miRNAs are usually located at fragile sites and genomic regions associated with tumors [2]. A large number of studies have shown that miRNA is related to the occurrence and development of various tumors, and is expected to become an important indicator for tumor diagnosis, treatment and prognosis evaluation [3]. Microrna-448 (Mir-448) was first found in bovine alveolar macrophages, and was speculated to be closely related to cell immune response, proliferation and apoptosis [4]. In recent years, there are increasing reports on Mir-448 at home and abroad. The relationship between Mir-448 and tumor was first reported in China after azithromycin chemotherapy for breast cancer, and it was found that azithromycin chemotherapy for breast cancer caused Mir-448 in breast cancer cells is significantly down-regulated, leading to up-regulated expression of SATB1, the target gene of Mir-448, resulting in increased expression of Twistl and activation of NF-KB signaling pathway, which ultimately leads to chemotherapy-induced epithelial-mesenchymal transformation of tumor cells [5]. Studies have shown that Mir-448 plays an important role in the occurrence and development of breast cancer, bladder cancer, osteosarcoma, oral squamous cell carcinoma and other diseases [6] [7] [8] [9], but the role of Mir-448 in colon cancer remains unclear. The purpose of this study was to investigate the role of Mir-448 in the development and progression of colon cancer and its potential molecular mechanism.

\section{Concept and Function of miRNA}

MicroRNAs are a class of endogenous non-coding Rnas with regulatory functions that widely exist in eukaryotic cells and are 20 - 25 nucleotides long. Lee and Ambros [10] first discovered MicroRNA in Caenorhabditis elegans. So far, more and more microRNAs have been discovered, and the functions and mechanisms of microRNAs have been gradually revealed. MicroRNA is highly conserved by species, expression sequence and tissue specificity, and plays a variety of roles in regulating cell growth and development [11]. Mature MicroRNAs first assemble RNA-induced silencing complexes, and then recognize target mrnas through base complementation, thereby regulating the expression of target mrnas and participating in the physiological and pathological processes of various cells [12]. Carcinogenesis is an extremely complex process involving multiple genes and steps. The main molecular mechanisms include activation and overexpression of proto-oncogenes and mutation and loss of tumor suppressor genes. At present, more and more evidences show that abnormally expressed MicroRNAs are closely related to the occurrence and development of tumors, and different tumors have different MicroRNAs expression characteristic profiles. Yan et al. [13] systematically revealed the expression of various MicroRNAs in tumor cells through large-scale parallel sequencing technology, and found the differential expression and its changing trend. Thurnherr et al. [14] conducted a further study to determine the specific expression of MicroRNA in liver cancer tissues and further explore its target genes. MicroRNA plays a key 
role in tumor metastasis and recurrence, and its mechanism may be related to cell cycle, DNA damage and repair, and apoptosis.

\section{Role of mirNA-448}

mirNA-448 is a $22 \mathrm{nt}$ length miRNA located at (idea) X-NC_000023.11 with a base sequence from 5' to 3' of UUGCAUAUGUAGGAUGUCCCAU. Mir-448 has been confirmed to be involved in the occurrence and development of a variety of malignant tumors, and to play a role of oncoprogenitor or oncosuppressor gene. In recent years, scholars at home and abroad have paid more and more attention to the study of mirNA-448, and found that mirNA-448 is involved in the regulation of cell proliferation, differentiation, apoptosis and other cell activities, and plays a very important role in the development and growth of the body, the onset and progression of some diseases and the occurrence and metastasis of tumors.

\subsection{The Role of mirNA-448 in Regulating Adipose Tissue}

Many miRNAs are involved in the regulation and expression of serotonin (5-HT) transporters in the brain and other tissues. Serotonin acts as a channel for communication and information transmission between certain nerves. 5-HT also regulates the activity of some specific miRNAs. Studies [15] have found that 5-HT2C receptor and mirNA-448 (located in the intra region of the fourth sequence of 5-HT2C) control the process of adipocyte differentiation and interact with each other. mirNA-448 and 5-HT2C receptor regulate adipocyte differentiation respectively, and the density of 5-HT2C receptor is upregulated during adipocyte differentiation. This causes 5-HT2C receptors to stimulate fat cells, which may contribute to obesity by increasing the number and swelling of fat cells. At the same time, mirNA-448 was transcribed simultaneously with 5-HT2C to reduce adipocyte differentiation, suggesting that obesity is well regulated by the same gene sequence. The regulation mechanism of 5-HT on adipocytes is not clear. mirNA-448 regulates the inhibitory transcription factor K1f5, and the overexpression of mirNA-448 reduces the expression of Klf5, which in turn reduces the expression of adipocytes and the accumulation of triglycerides. In the development of the body, mirNA-448, as a microrNA regulating obesity, plays a very important role, which is worthy of further study and provides a new method for studying the causes and treatment of human obesity. Meanwhile, KLF5 expression is regulated by different cancers, and it also controls the survival, proliferation, apoptosis and metastasis of cancer cells. Its regulation in gliomas and other tumor types may be related to the interaction between mirNA-448 and other miRNAs and the influence of 5-HT2B. Therefore, mirNA-448 May also play an important role in a variety of tumors. mirNA-448 has not been found to play a role in the development of other tissues, but mirNA-448 is highly likely to play a potential role in the process of human development, which requires our efforts to further study. 


\subsection{The Role of mirNA-448 in the Pathogenesis and Progression of Disease}

Some miRNAs play an important role in the process of virus infection, and some miRNAs affect the process of virus infection by regulating immune factors in human body. Innate immunity is a natural immune barrier gradually formed during the long period of phylogenetic development and evolution. It is the first barrier to resist the invasion of pathogenic microorganisms. Studies have shown that miRNA may be involved in multiple response links of innate immunity in vertebrates. When pathogenic microorganisms are infected, miRNA is not only an important signal regulation molecule after activation of innate immune receptors, but also directly interferes with viral replication to exert viral effects [16]. Viruses can also directly or indirectly interfere with the expression of many host immune-related genes through self-encoded miRNA or changing the expression profile of miRNA in host cells to achieve the purpose of escaping immune clearance. $\mathrm{Xu}$ et al. [17] cloned and identified 22 miRNAs from bovine alveolar macrophages, among which 8 were BTA-mirNA-448, which were not recognized in bovine lungs but were directly homologous to miRNAs in humans and mice. It was found that mirNA-448 may be closely associated with cell-mediated immune response against intracellular bacterial toxicity in bovine lung. Many miRNAs are involved in the process of some viral infections, and HCV RNA replication is associated with many miRNAs. Studies have shown that mirNA-448 can directly block HCV replication, and mirNA-448 has a direct target in HCV RNA, located at the core coding sequence of 801. Overexpressed mirNA-448 can reduce HCV replication [18]. Pedersen et al. [19] also found that Mir-NA-448 is expressed in bovine alveolar macrophages and has the ability to block hepatitis $\mathrm{C}$ virus (HCV) replication, which is associated with cell-mediated immune response. Conrad et al. [20] found in their study on HCV RNA that at least five microRNAs are involved in the blocking of HCV RNA replication, and mirNA-448 is one of them. Therefore, mirNA-448 can play an important role in the prevention, treatment and diagnosis of viral infection as a potential method, and the addition of mirNA-488 therapy in the treatment of HCV may play a positive role in supporting the treatment of the disease. However, the proteins involved in the molecular mechanism of mirNA-448's role in HCV replication and the interaction between mirNA-448 and protein are still unknown, which are awaiting further study.

miRNA has been found to play an important role in normal brain, neurological diseases and neurotoxicity, and mirNA-448 also plays a unique role in the nervous system. An et al. [21] used microarray and QRT-PCR techniques to study the miRNA of hippocampus in mouse models of chronic neurotoxicity under lead exposure, and found that mirNA-448, mirNA-204, mirNA-211, mirNA-449a, mirNA-34B and mirNA-34C increased significantly. mirNA-494 was significantly down-regulated. Among them, mirNA-448 had the highest up-regulation ratio, which reached 30 times compared with normal non-exposed 
control mice. These miRNA-related target genes were analyzed by three bioinformatics databases, and further confirmed by QRT-PCR and Western Blot. Six up-regulated miRNAs, including mirNA-448, were found to be associated with nerve injury, neurodegeneration, axonal and synaptic functions, and nerve development and regeneration, all of which are the main targets of lead toxicity. The mature mRNA expression level and protein target genes (Bcl-2, Itprl, Map2k1) are significantly inhibited, and the disorder of mirNA-448 may subsequently regulate downstream target genes, resulting in the disorder of homeostasis in the brain, abnormal synapses, impaired learning and memory, and neural degeneration. However, the specific mechanism is still unclear and needs further study.

\subsection{The Role of mirNA-448 in Tumor Genesis and Metastasis}

Hepatocellular carcinoma (HCC) is one of the most common cancers, with a high probability of postoperative recurrence and metastasis and a low five-year survival rate. The treatment of HCC is one of the most urgent medical problems in the world. The abnormal expression of mirNA-448 in HCC may be closely related to the occurrence and development of HCC. Katayama et al. [22] prepared miRNA expression profiles expressed in cancer by studying miRNA expression in tumor and non-tumor tissues of 40 groups of HCC patients and surrounding tissues of 6 groups of metastatic HCC patients. Twelve were infected with hepatitis $\mathrm{C}$ virus (HCV); sixteen patients were not infected with HBV or HCV. Six HCC with colon metastasis were used as control samples. The results showed that mirNA-448 was significantly down-regulated in tumor tissues and was not affected by liver disease infection. These results indicate that mirNA-448 May be a tumor suppressor gene for HCC, and can be used as a biological indicator of HCC unaffected by viral infection, providing a potential biological marker for the diagnosis and prognosis of HCC. However, the specific role of mirNA-448 in HCC still needs to be further verified, and it is of great research value. The regulatory feedback loop of NF-KB/mirNA-448 plays an important role in epithelial-mesenchymal transformation induced by chemotherapy in breast cancer. Morphological changes involved in the transformation of tumor cells from non-invasive to malignant phenotypes are called epithelial-mesenchyal transition (EMT) [23]. Some literatures indicate that EMT is closely related to the sensitivity of chemotherapy drugs. Tumor cells undergoing EMT significantly increased the ability of metastasis, invasion and multidrug resistance. Li [24] was cultured in the presence of adriamycin.

The expression changes of 113 different mirNAS were analyzed in MCF7 cells, which were cultured for 36 hours. The most obvious change was mirNA-448, which was down-regulated by QRT-PCR. In addition, compared with mesenchymal tumors, the inhibition of miRNA 448 in epithelial tumor cells was most obvious after adriamycin treatment. At the same time, mirNA-448 regulates SATB1 through the AR-EGFR channel, and inhibition of mirNA-448 directly 
promotes the expression of specific anticoagulase sequence-binding protein-1 (SATB1), thereby enhancing the expression of dimodin and epidermal growth factor receptor (EG-FR) regulated Twistl, thus inducing the generation of EMT. Meanwhile, it also stimulates the activity of nuclear factor KB (NF-KB). Through the analysis of Matlnspeetoi software, mirNA-448 was predicted and confirmed to be the transcription target of nuclear factor $\mathrm{KB}$, indicating that adriamycin activated NF-KB directly inhibited the transcription of mirNA-448 promoter to achieve the transcription inhibition of mirNA-448. A positive feedback loop was formed between NF-KB and mirNA-448. Chemotherapy is very common in the clinical treatment of tumors, but it will destroy both normal cells and tumor cells at the same time, and will bring great side effects to patients in the treatment of tumors. EMT is one of many side effects after chemotherapy [25]. Therefore, the destruction of the positive feedback loop between NF-KB and mirNA-448 in the clinical treatment of breast cancer chemotherapy may reduce the production of EMT and improve the therapeutic effect of prognosis. The combined use of drugs targeting mirNA-448 may improve the efficacy of systematic treatment for breast cancer patients, which is worthy of further exploration and research.

\section{Prospects}

So far, many microRNAs and their target genes have not been discovered, and the composition of their regulatory network has not been thoroughly studied. Through recent studies, it has been found that mirNA-448 can affect the development and growth of the body, the onset and progression of some diseases, and the occurrence and metastasis of tumors. In addition, the regulatory mechanism of mirNA-448 related signaling pathway has been preliminarily proved, which indicates that mirNA-448 is an important and multipotent miRNA. Such small molecules have a wide range of regulatory functions in life activities, and have profound and complex effects on gene expression, growth and development, and behavior. However, further research is needed in many details. The research on mirNA-448 is still seriously inadequate, and many potential functions have not been discovered.

\section{Foundation Project}

Supported by Hainan Natural Science Foundation (Project No. 821MS060).

\section{Conflicts of Interest}

The authors declare no conflicts of interest regarding the publication of this paper.

\section{References}

[1] Wang, Z. (2007) Bionomics and Behavior of the Wolf Spider, Pardosa pseudoannulata (Araneae: Lycosidae). Acta Entomologica Sinica, 50, 927-932.

[2] Ma, J. and Zhang, S.C. (2012) Structure and Function of Vitelloprotein. Journal of 
Ludong University (Natural Science Edition), 28, 252-260.

[3] Avarre, J.C., Lubzens, E. and Babin, P. (2007) Apolipocrustacein, Formerly Vitellogenin, Is the Major Egg Yolk Precursor Protein in Decapod Crustaceans and Is Homologous to Insect Apolipophorin II/I and Vertebrate Apolipoprotein B. BMC Evolutionary Biology, 7, 3. https://doi.org/10.1186/1471-2148-7-3

[4] Lv, Y., Lei, Y., Hu, W., Ding, C., Zhang, C. and Fang, C. (2015) miR-448 Negatively Regulates Ovarian Cancer Cell Growth and Metastasis by Targeting CXCL12. Clinical \& Translational Oncology. Official Publication of the Federation of Spanish Oncology Societies and of the National Cancer Institute of Mexico, 17, 903-909. https://doi.org/10.1007/s12094-015-1325-8

[5] Shan, C.T., Fei, F., Li, F.Z., Zhuang, B., Zheng, Y.L., Wan, Y.F. and Chen, J.H. (2017) miR-448 Is a Novel Prognostic Factor of Lung Squamous Cell Carcinoma and Regulates Cells Growth and Metastasis by Targeting DCLK1. Biomedicine \& Pharmacotherapy, 89, 1227-1234. https://doi.org/10.1016/j.biopha.2017.02.017

[6] Lee, S. and Jiang, X. (2017) Modeling miRNA-mRNA Interactions That Causephenotypic Abnormality in Breast Cancer Patients. PLoS ONE, 12, e0182666. https://doi.org/10.1371/journal.pone.0182666

[7] Cheng, Y., Yang, X., Deng, X., et al. (2015) MicroRNA-218 Inhibits Bladder Cancer Cell Proliferation, Migration, and Invasion by Targeting BMI-1. Tumor Biology, 36, 8015-8023. https://doi.org/10.1007/s13277-015-3532-X

[8] Wu, X., Yan, L., Liu, Y., et al. (2017) MicroRNA-448 Suppresses Osteosarcoma Cell Proliferation and Invasion through Targeting EPHA7. PLOS ONE, 12, e0175553. https://doi.org/10.1371/journal.pone.0175553

[9] Shen, L., Liu, L., Ge, L., et al. (2016) miR-448 Downregulates MPPED2 to Promote Cancer Proliferation and Inhibit Apoptosis in Oral Squamous Cell Carcinoma. EXperimental and Therapeutic Medicine, 12, 2747-2752. https://doi.org/10.3892/etm.2016.3659

[10] Lee, R.C. and Ambros, V. (2001) An Extensive Class of Small RNAs in Caenorhabditis elegans. Science, 294, 862-864. https://doi.org/10.1126/science.1065329

[11] Ambros, V. (2004) The Functions of Animal microRNAs. Nature, 431, 350-355. https://doi.org/10.1038/nature02871

[12] Kim, V.N. and Nam, J.W. (2006) Genomics of microRNA. Trends in Genetics, 22, 165-173. https://doi.org/10.1016/j.tig.2006.01.003

[13] Yan, C., Shi, X., Wang, Q., et al. (2014) Genome-Wide Identification and Expression Analysis of microRNA Involved in Small Cell Lung Cancer via Deep Sequencing. Molecular Medicine Reports, 10, 2633-2642.

https://doi.org/10.3892/mmr.2014.2535

[14] Thurnherr, T., Mah, W.C., Lei, Z., et al. (2016) Differentially Expressed MicroRNAs in Hepatocellular Carcinoma Target Genes in the Genetic Information Processing and Metabolism Pathways. Scientific Reports, 6, Article No. 20065. https://doi.org/10.1038/srep20065

[15] Kinoshita, M., Ono, K., Horie, T., et al. (2010) Regulation of Adipocyte Differentiation by Activation of Serotonin (5-HT) Receptors 5-HT2AR and 5-HT2CR and Involvement of miRNA-448 Mediated Repression of Klf5. Endocrinology, 24, 1978-1987. https://doi.org/10.1210/me.2010-0054

[16] Hou, Z.H., Zhang, J. and Tian, Z.G. (2008) Molecular Mechanism of MicroRNA Regulation of Innate Immune Response. Progress in Biochemistry and Biophysics, 35, 1131-1136. 
[17] Xu, G., Zhang, Y., Jia, H., et al. (2009) Cloning and Identification of microRNAs in Bovine Alveolar Macrophages. Molecular and Cellular Biochemistry, 332, 9-16. https://doi.org/10.1007/s11010-009-0168-4

[18] Pedersen, I.M., Cheng, G., Wieland, S., et al. (2007) Interferon Modulation of Cellular microRNAs as an Antiviral Mechanism. Nature, 449, 919-922. https://doi.org/10.1038/nature06205

[19] Pedersen, I. and David, M. (2008) MicroRNAs in the Immune Response. Cytokine, 43, 391-394. https://doi.org/10.1016/j.cyto.2008.07.016

[20] Conrad, K.D. and Niepmann, M. (2014) The Role of microRNAs in Hepatitis C Virus RNA Replication. Archives of Virology, 159, 849-862.

https://doi.org/10.1007/s00705-013-1883-4

[21] An, J., Cai, T., Che, H., et al. (2014) The Changes of miRNA Expression in Rat Hippocampus Following Chronic Lead Exposure. Toxicology Letters, 229, 158-166. https://doi.org/10.1016/j.toxlet.2014.06.002

[22] Katayama, Y., Maeda, M., Miyaguchi, K., et al. (2012) Identification of Pathogenesis-Related microRNAs in Hepatocellular Carcinoma by Expression Profiling. Oncology Letters, 4, 817-823. https://doi.org/10.3892/ol.2012.810

[23] Huber, M.A., Kraut, N. and Beug, H. (2005) Molecular Requirements for Epithelial-Mesenchymal Transition during Tumor Progression. Current Opinion in Cell Biology, 17, 111. https://doi.org/10.1016/j.ceb.2005.08.001

[24] Li, Q.Q., Chen, Z.Q., Cao, X.X., et al. (2011) Involvement of NF-kappaB/miR-448 Regulatory Feedback Loop in Chemotherapy-Induced Epithelial, Mesenchymal Transition of Breast Cancer Cells. Cell Death \& Differentiation, 18, 16-25. https://doi.org/10.1038/cdd.2010.103

[25] Liu, S.Y., Xie, L., Qi, B., et al. (2014) Study on the Expression Profile of Differential microrNA/mRNA in Oral Squamous Cell Carcinoma. West China Journal of Stomatology, 32, 400-403. 